\title{
Effect of image-guided hypofractionated stereotactic radiotherapy on peripheral non-small-cell lung cancer
}

This article was published in the following Dove Press journal:

OncoTargets and Therapy

16 August 2016

Number of times this article has been viewed

\author{
Shu-wen Wang' \\ Juan Ren' \\ Yan-li Yan ${ }^{2}$ \\ Chao-fan Xue ${ }^{2}$ \\ $\mathrm{Li} \operatorname{Tan}^{2}$ \\ Xiao-wei $\mathrm{Ma}^{2}$ \\ 'Department of Radiotherapy, First \\ Affiliated Hospital of Xian Jiaotong \\ University, ${ }^{2}$ Medical School of Xian \\ Jiaotong University, Xi'an, Shaanxi, \\ People's Republic of China
}

Correspondence: Juan Ren Department of Radiotherapy, First Affiliated Hospital of Xian Jiaotong University, 277 Yan Ta Xi Lu, Xi'an, Shaanxi 71006I,

People's Republic of China

Tel +86298532 4029

Email869491533@qq.com
Abstract: The objective of this study was to compare the effects of image-guided hypofractionated radiotherapy and conventional fractionated radiotherapy on non-small-cell lung cancer (NSCLC). Fifty stage- and age-matched cases with NSCLC were randomly divided into two groups (A and B). There were 23 cases in group A and 27 cases in group B. Image-guided radiotherapy (IGRT) and stereotactic radiotherapy were conjugately applied to the patients in group A. Group A patients underwent hypofractionated radiotherapy (6-8 Gy/time) three times per week, with a total dose of 64-66 Gy; group B received conventional fractionated radiotherapy, with a total dose of 68-70 Gy five times per week. In group A, 1-year and 2-year local failure survival rate and 1-year local failure-free survival rate were significantly higher than in group $\mathrm{B}(P<0.05)$. The local failure rate $(P<0.05)$ and distant metastasis rate $(P>0.05)$ were lower in group A than in group B. The overall survival rate of group A was significantly higher than that of group B $(P=0.03)$, and the survival rate at 1 year was $87 \%$ vs $63 \%,(P<0.05)$. The median survival time of group A was longer than that of group B. There was no significant difference in the incidence of complications between the two groups $(P>0.05)$. Compared with conventional fractionated radiation therapy, image-guided hypofractionated stereotactic radiotherapy in NSCLC received better treatment efficacy and showed good tolerability.

Keywords: non-small-cell lung cancer, hypofractionated radiotherapy, stereotactic radiotherapy, segmentation, intensity-modulated radiotherapy, image-guided radiation therapy technology

\section{Introduction}

Lung cancer is one of the malignant tumors with the highest mortality in the People's Republic of China. For patients suffering from inoperable non-small-cell lung cancer (NSCLC), radiotherapy is the main treatment measure. The treatment efficacy of lung cancer is still not satisficatory, and the 5-year overall survival (OS) rate is $\leq 15 \%$. Although the treatment efficacy in early-stage NSCLC patients can be improved by radiotherapy, the 5 -year OS rate is only $5 \%-42 \%$.

The local control rate of lesion is relatively associated with the dose of radiotherapy. Different segmentation doses of radiotherapy have different effects on the treatment outcome. In theory, the higher the radiotherapy dose, the stronger is the killing effect on tumor cells. Hence, it is good to increase the dose segmentation and shorten the total treatment time.

Hypofractionated stereotactic radiotherapy is capable of delivering maximal radiation dose to the tumor tissue, while minimizing the dose to the surrounding normal tissue. Hypofractionated radiotherapy can improve the biological effective dose to block the proliferation of tumor cells and shorten the whole treatment time. This technique 
can also decrease the regeneration time of tumor cells by significantly shortening the total treatment time. The regeneration of NSCLC cells occurs rapidly, with a doubling time of 2.5-3.3 days, while the treatment time spans more than 6 weeks. When the treatment time is extended by 1 day, the survival rate will reduce by $1.6 \% .^{1,2}$ Image-guided radiotherapy (IGRT) technology is an accurate radiotherapy technology developed in recent years. ${ }^{3}$ Hypofractionated irradiation is made possible by IGRT technique through checking and adjusting the treatment errors in real time.

In order to compare the effects of the image-guided hypofractionated stereotactic radiotherapy and conventional radiotherapy in NSCLC, cone beam computed tomography (CT) and image-guided stereotactic radiotherapy were conducted in this randomized study. The differences in the efficacy and toxicity between the conventional segmentation and hypofractionated stereotactic radiotherapy were compared.

\section{Materials and methods}

Fifty cases of NSCLC patients who were not suitable for surgery, or who refused surgery, were selected from January 2010 to February 2016. Thirty-six cases were male and 14 cases were female, with ages ranging from 33 to 80 years. The pathological diagnosis was made through fiberoptic bronchoscopy or percutaneous needle biopsy. According to tumor node metastasis (TNM) staging criteria, nine cases were stage IA, seven cases were stage IB, five patients were stage IIB, five patients were stage IIIA, 13 patients were stage IIIB, and eleven patients were stage IV. Among them, 26 cases were squamous cell carcinoma, 17 cases were adenocarcinoma, one case was large cell carcinoma, and the pathology of six cases was unclear. All 50 cases were peripheral lung cancers and had not received any radiotherapy before. Karnofsky score for all patients was $\geq 80$. The functions of liver and kidney and the hematopoietic functions of the bone marrow were normal. The patients were randomly divided into two groups. Twentythree cases were included in the hypofractionated group and 27 cases in the conventional fractionated group (Table 1). The study was formally approved by the Ethic Committees of the First Hospital Affiliated to Xi'an Jiao Tong University. Written informed consent was obtained from the patients.

\section{Radiation therapy}

Radiation therapy was given using Elekta Synergy medical linear accelerator. The position of each patient's body was fixed by body positioning phantom. CT simulation was performed by enhanced CT scanning with patients breathing quietly. Patients were scanned from the thoracic entrance to the level of the costophrenic angle, with a $5 \mathrm{~mm}$ scanning thickness. The scanned electronic images were transmitted to the treatment planning system. Tumor target volume was delineated by the radiotherapy and imaging physicians together in accordance with the standard definition stipulated in Report $50^{4}$ and Report $62^{5}$ documents of the International Radiation Units and Measurement Committee.

Gross target volume (GTV) is the entire tumor area detected by clinical and radiographic examination, including gross target volume-primary tumor (GTV-P) and including gross target volume-regional metastasis lymph node (GTV-N). Lymph nodes with a diameter greater than $1 \mathrm{~cm}$

Table I Characteristics of the patients $(n=50)$

\begin{tabular}{lll}
\hline Characteristics & Hypofractionated group & Conventional fractionated group \\
\hline Age (years) & & $33-80$ \\
Range & $49-80$ & 66 \\
Median & 68 & 22 \\
Sex & 14 & 5 \\
Male & 9 & 2 \\
Female & 7 & 3 \\
Disease staging & 4 & 0 \\
la & 0 & 4 \\
Ib & 1 & 4 \\
Illa & 1 & 12 \\
Ilb & 1 & 2 \\
IIla & 9 & Squamous cell carcinoma (I8) \\
IIIb & Squamous cell carcinoma (8) & Adenocarcinoma (8) \\
IV & Adenocarcinoma (9) & Unclear (I) \\
Pathology & Large cell carcinoma (I) & 27 \\
& Unclear (5) & 27 \\
\hline
\end{tabular}


in the CT scan were judged as positive lymph node. GTV-P was delineated in lung window setting while GTV-N was delineated in mediastinal window setting. The clinical target volume was judged based on the size of tumor and lymph node prior to chemotherapy.

The planning target volume was determined based on the position error and the patient's respiratory motion. The field direction, the field weight, and the field fraction were designed through the Beam-field Equation Vision (BEV) and Reaction Equation Vision (REV).

$\mathrm{X}$-ray examination was performed on a weekly basis during the treatment and was compared with the simulated images and digitally reconstructed images to determine the accuracy of target area and the patient position. Group A patients underwent hypofractionated radiotherapy with 6-8 Gy/time, once every other day, three times per week, with a total dose of 64-66 Gy. Group B patients received conventional fractionated radiotherapy, with a total dose of 68-70 Gy.

\section{Stereotactic radiotherapy plan}

Stereotactic radiotherapy plans included intensity-modulated radiation therapy (IMRT) and volumetric-modulated arc therapy (VMAT) plans. The treatment plan is selected based on whether this plan is capable or not of achieving a better target coverage, and organs at risk protection.

\section{IMRT plan}

The IMRT optimization was performed by applying a direct machine parameter optimization (DMPO) algorithm in our treatment planning system (Pinnacle3; Philips Radiation Oncology Systems, Fitchburg, WI, USA), as described previously. ${ }^{6}$ For each plan, five or seven coplanar beams were used depending on the tumor location. In the plan generation, maximum iterations and maximum number of segments in the plan optimization were 50 and 80 , respectively, and the maximum MUs and segment area were $5 \mathrm{MU}$ and $5 \mathrm{~cm}^{2}$, respectively. Plans were generated for the Elekta Beam Modulator with 10 MV X-ray beams (Figure 1).

\section{VMAT plan}

The VMAT planning was done by applying the SmartArc planning algorithm in Pinnacle3 version 9.2 (research version, Philips Radiation Oncology Systems). Single or dual arcs were employed depending on the tumor location.
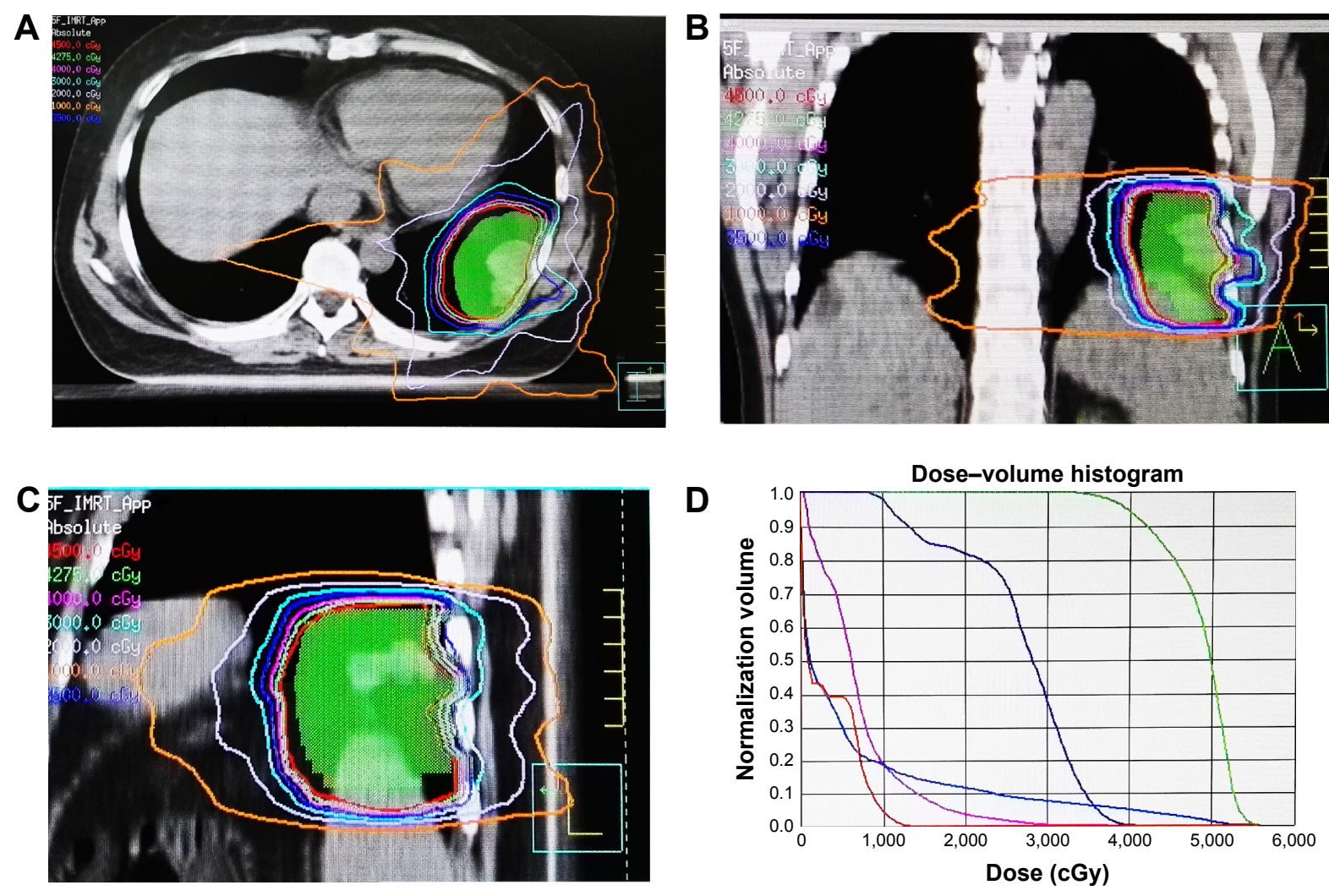

Figure I The distribution of isodose curve and dose-volume histograms of one of the IMRT plans.

Notes: (A-C) The distribution of isodose curve of one of the IMRT plans, $(\mathbf{A})$ in cross cutting image, $(\mathbf{B})$ in coronal image, and $(\mathbf{C})$ in sagittal image. The green shadow is PTV which includes the range-of-motion of the target lesion. (D) Dose-volume histograms from one of the IMRT plans.

Abbreviations: IMRT, intensity-modulated radiation therapy; PTV, planning target volume. 
The accelerator used automatic dose rate was chosen for each individual segment of the arc. Plans were generated with $10 \mathrm{MV}$ X-ray beams (Figure 2).

\section{Plan evaluation}

The quality of plans was evaluated by three radiation oncologists. Dose-volume histograms (DVHs) and the corresponding dose distributions of plans were independently reviewed by each oncologist. It was proposed that $95 \%$ of the target volume should be encompassed by $95 \%$ of the prescribed dose. Planning target volume coverage was evaluated by using $\mathrm{D}_{\text {max }}, \mathrm{D}_{\text {min }}, \mathrm{D}_{\text {mean }}$, the heterogeneity index (HI), and the conformity index $(\mathrm{CI}){ }^{7}$

$$
\mathrm{HI}=\frac{D_{2 \%}-D_{98 \%}}{D_{50 \%}}
$$

$D_{2 \%}, D_{98 \%}$, and $D_{50 \%}$ represent the dose for $2 \%, 98 \%$, and $50 \%$ target volume. Values of $\mathrm{HI}$ closer to 0 indicate greater dose homogeneity within the volume of planning target volume, while larger values indicate more heterogeneous dose distribution.
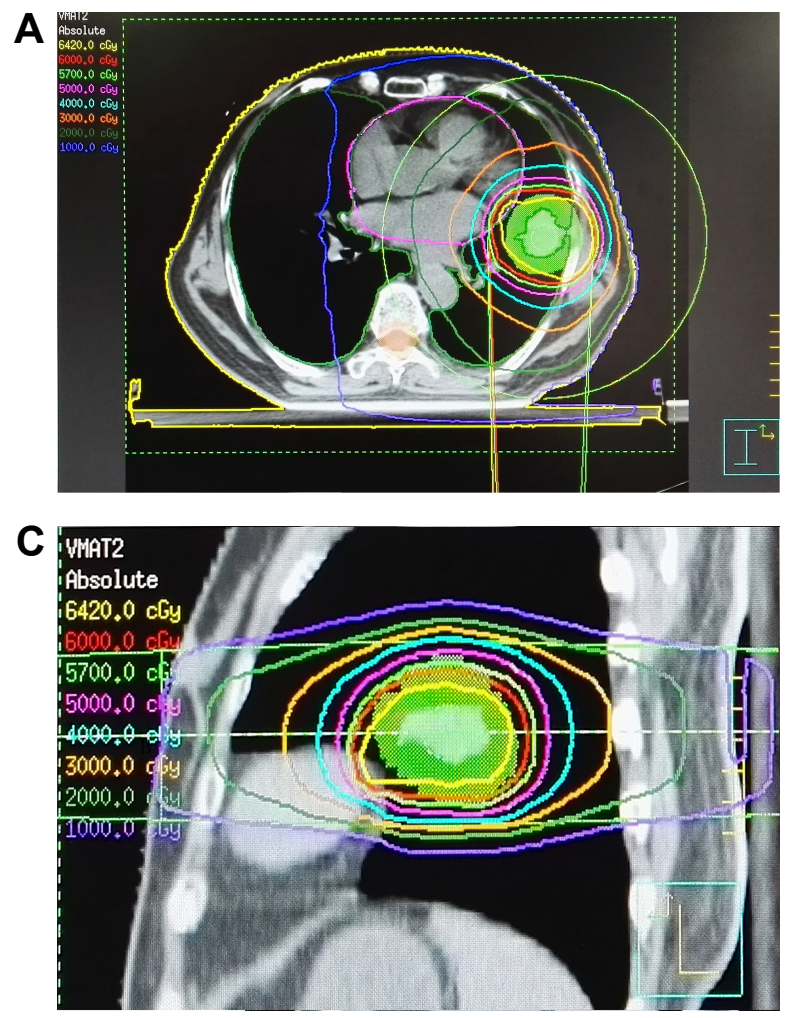

$$
\mathrm{CI}=\frac{V_{T, r e f}}{V_{T}} \times \frac{V_{T, r e f}}{V_{\text {ref }}}
$$

$V_{T, \text { ref }}=$ volume of target receiving a dose equal to or greater than the reference dose, $V_{T}=$ volume of target, $V_{\text {ref }}=$ volume receiving a dose equal to or greater than the reference dose (treated volume) ${ }^{8}$ The closer the value of $\mathrm{CI}$ is to 1.0 , the better would be the dose conformity.

The distribution of isodose curve and DVHs from one of the IMRT plans were randomly selected and are shown in Figure 1. The distribution of isodose curve and DVHs from one of the VMAT plans were randomly selected and shown in Figure 2.

\section{Images acquisition technology during the treatment}

Elekta Synergy ${ }^{\circledR}$ system (Elekta AB, Stockholm, Sweden) integrates the treatment accelerator with the image acquisition guiding system which is based on the principle of X-ray volume imaging. Synergy system is designed to provide three-dimensional (3D) X-ray volume imaging (XVI) with $\mathrm{kV}$ level. XVI is an advanced imaging system, which can
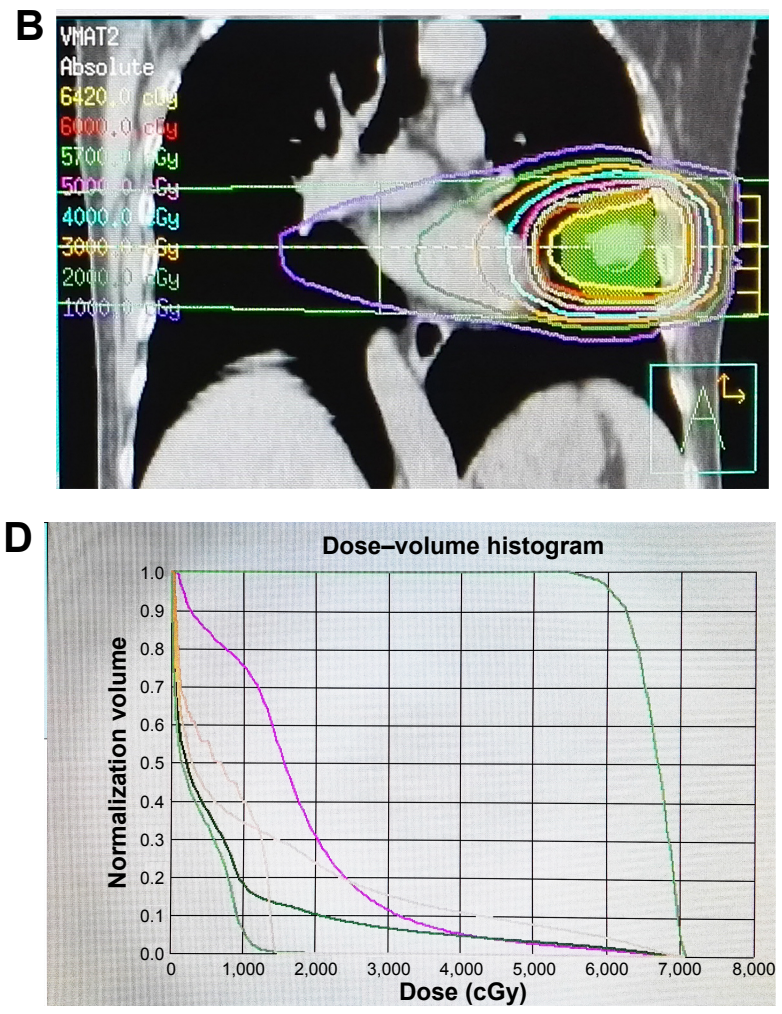

Figure 2 The distribution of isodose curve and dose-volume histograms from one of the VMAT plans.

Notes: $(\mathbf{A}-\mathbf{C})$ The distribution of isodose curve of one of the VMAT plans, $(\mathbf{A})$ in cross cutting image, (B) in coronal image, and $(\mathbf{C})$ in sagittal image. The green shadow is PTV which includes the range-of-motion of the target lesion. (D) Dose-volume histograms from one of the VMAT plans.

Abbreviations: VMAT, volumetric-modulated arc therapy; PTV, planning target volume. 
obtain two-dimensional (2D) and 3D kV-level images of treatment position during the treatment. XVI can use the image management tools to automatically and remotely correct the bed position.

The image guidance functions of Elekta Synergy ${ }^{\circledR}$ system include the function of obtaining the real-time images of accelerator using iViewGT. The PlanarView software supports the acquisition of static 2D planar high-quality $\mathrm{kV}$-level images. Under this image mode, the positioning mark can be clearly seen. The image processing tool supports the comparison of the collected 3D volumetric imaging data with the planning CT data, and also supports the online and offline adaptive radiotherapy technology (Figure 3).

\section{Error analysis and adjustment before and during the treatment}

The first XVI image, was obtained before the treatment. The acquired volume images and planning images were matched through the automatic matching function of the system, and the errors of the target center in the X, Y, Z directions were acquired and corrected. The second volume image was obtained after the error adjustment. The irradiation was implemented if the error was less than $2 \mathrm{~mm}$. The third XVI image, was obtained after the treatment. Matching images of four patients were randomly selected and are shown in Figure 3, which shows the image matching results during the treatment (Figure 3 ).

\section{Multileaf collimator system}

Multileaf collimator equipment of Elekta Synergy ${ }^{\circledR}$ system is a full built-in integrated fine field forming system, providing an accurate collimator system used universally for the $3 \mathrm{D}$ radiotherapy and accurate IMRT technology. Irradiation field of the small multileaf system comprises 80 independently controlled blades and the field size is $16 \times 21 \mathrm{~cm}$. The trip distance of every blade is more than $21 \mathrm{~cm}$. Since the thickness of the blade is $0.4 \mathrm{~cm}$ (at the isocenter), the blade can form the "fork finger" and the relative blades insert into each other's slots. The little multileaf can form many little fields in one field in one step.

\section{Evaluation of therapeutic efficiency}

During radiotherapy, the acute radiation reaction, hemogram, and Karnofsky score were evaluated on a weekly basis. Radiation injury was evaluated according to the Radiation Therapy Oncology Group (RTOG) acute radiation injury standard and the RTOG/European Organisation for Research and Treatment of Cancer (EORTC), late radiation injury classification scheme monthly during the first 6 months after the radiotherapy. After the first 6 months, patients were followed up every 3 months. The OS rate, cause-specific survival rate, recurrence-free survival rate, local recurrence, and distant metastasis were recorded and calculated.

\section{Statistics}

SPSS18.0 statistical software (SPSS Inc., Chicago, IL, USA) was used for statistics analysis. OS rate, local failure-free survival rate (LFFS), and local failure survival rate (LFS) curves were constructed using the Kaplan-Meier method. Log-rank method was employed to compare the survival rate between the groups. The survival rate at a certain time point was compared with $Z$-test, $Z>1.96$ was considered to be statistically significant. Local failure rate and distant metastasis rate were tested by chi-square test. $P \leq 0.05$ was considered as statistical significance. Position errors before correction, after correction, and after treatment were compared using one-way analysis of variance (ANOVA).

\section{Results}

\section{Treatment effect}

Two groups of patients were followed up for 4-61 months (average 32.5 months) to compare the treatment effect. A total of 33 patients died, all of them from cancer. One-year OS rate of the hypofractionated group (group A) was significantly higher than that of conventional fractionated group (group B) $(87.0 \%$ vs $63.0 \%, P<0.05)$, and 2-year OS rate of group A was also higher than that of group B (59.5\% vs $50.6 \%, P>0.05$ ) (Figure 4).

One-year LFS of group A was significantly higher than that of group B $(95.2 \%$ vs $72.8 \%, P<0.05)$, and 2 -year LFS of group A was also higher than that of group B (88.9\% vs $48.5 \%, P<0.05$ ) (Figure 5).

One-year LFFS of group A was significantly higher than that of group B $(87.0 \%$ vs $57.8 \%, P<0.05)$, and 2 -year LFFS of group A was also higher than that of group B (53.9\% vs $47.3 \%, P>0.05$ ) (Figure 6).

Local failure rate $(13.0 \%$ vs $40.7 \%, P<0.05)$ and Distant metastasis rate $(21.7 \%$ vs $44.4 \%, P>0.05)$ of Group A were all lower than that of Group B.

The median survival time of groups A and B were 34 and 17.5 months, respectively (Table 2).

\section{Detection and correction of errors}

Errors of the target center were monitored at the time of positioning before the treatment and compared with the planning target center in three directions: left-right direction, anteriorposterior direction, and cranial-caudal direction. The errors 


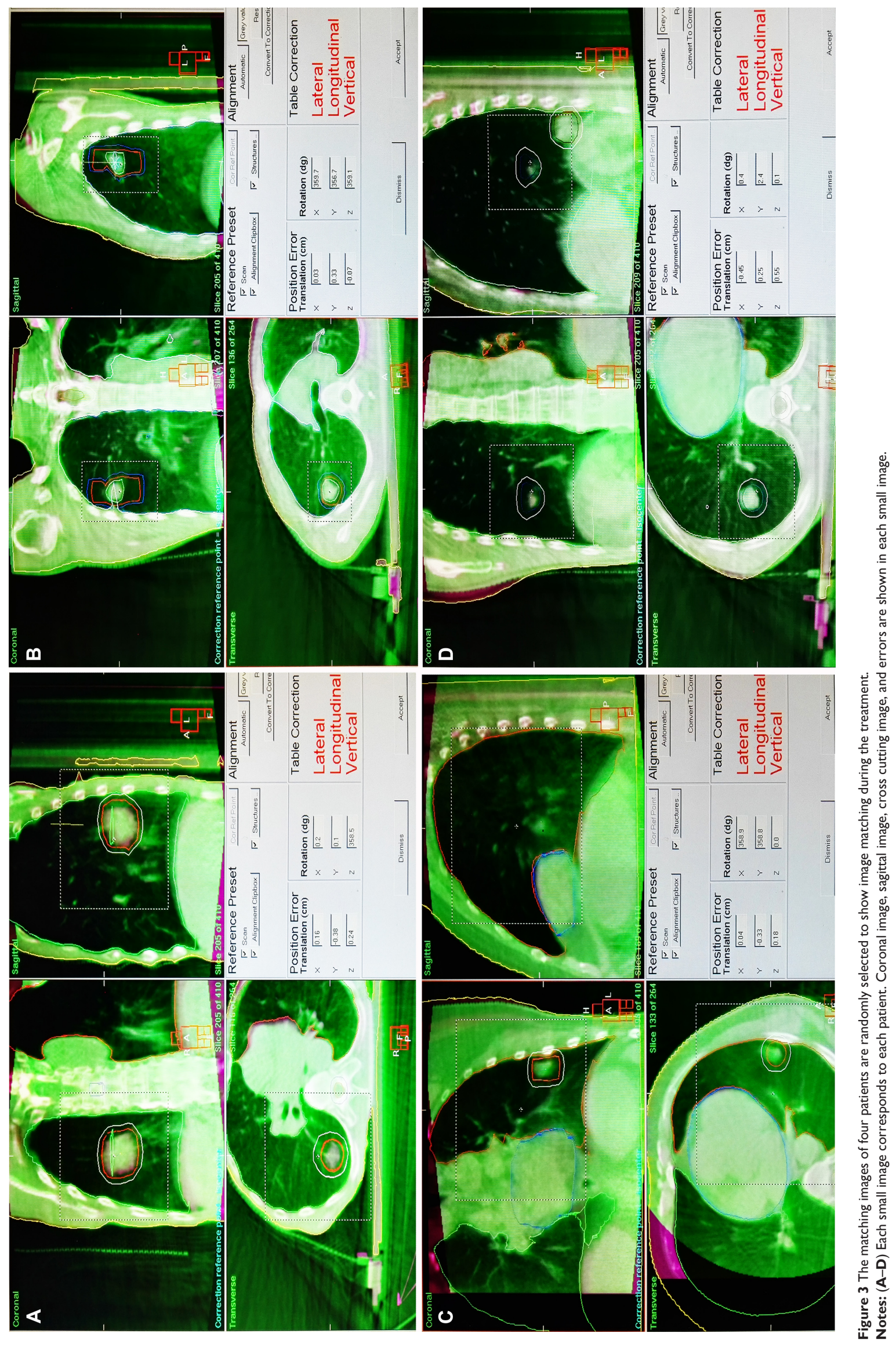




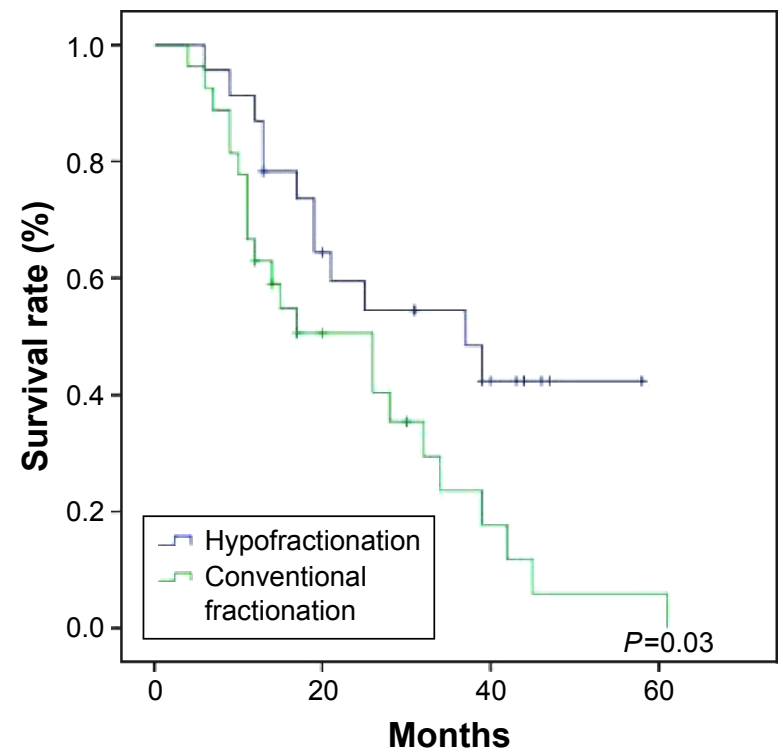

Figure 4 Overall survival rate curve.

Notes: Overall survival rate curve was constructed using the Kaplan-Meier method. One-year overall survival (OS) rate of hypofractionated group was significantly higher than that of conventional fractionated group $(P<0.05)$, and 2-year OS of group $A$ was also higher than that of group $B(P>0.05)$.

are shown in Table 3. The errors decreased after the correction and treatment, but there were no statistical differences between the errors that were measured before the adjustment, after the adjustment, and after the treatment (Table 3 ).

\section{Radiotherapy-related adverse reactions}

The radiotherapy-related adverse reactions were compared between the two groups. The adverse reactions including

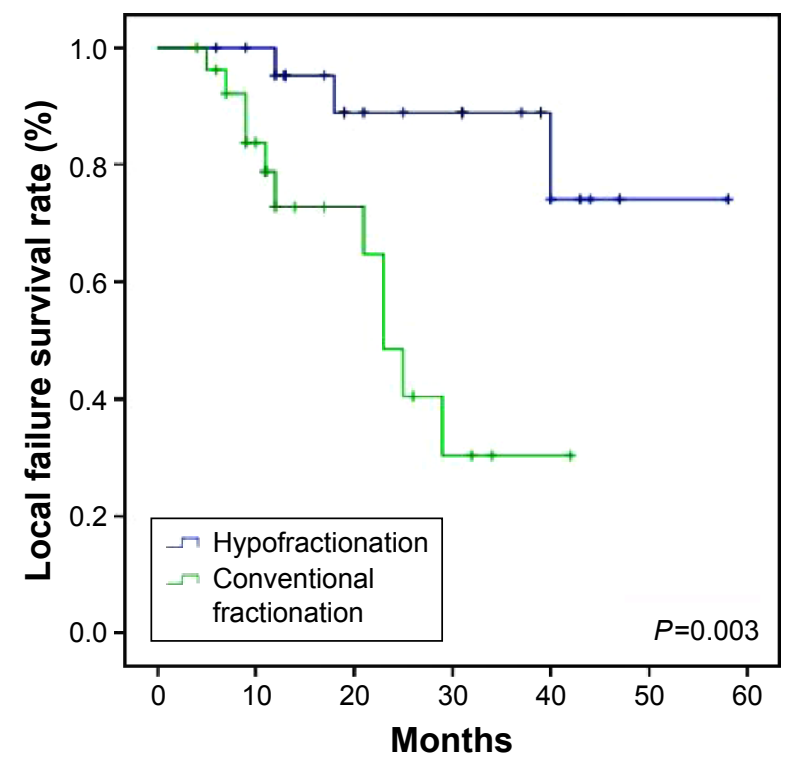

Figure 5 Local failure survival rate curve.

Notes: Local failure survival rate curve was constructed using the Kaplan-Meier method. One-year and 2-year local failure survival rates of group $A$ were significantly higher than those of group $B(P<0.05)$.

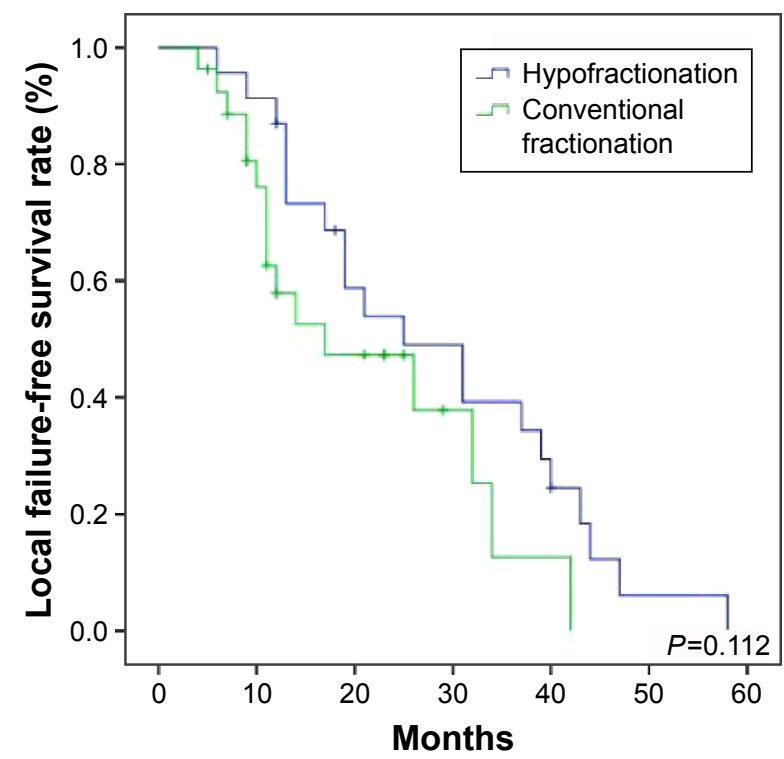

Figure 6 Local failure-free survival rate curve.

Notes: Local failure-free survival rate (LFFS) curve was constructed using the Kaplan-Meier method. One-year LFFS of group A was significantly higher than that of group $B(P<0.05)$. Two-year LFFS of group $A$ was also higher than that of group $B(P>0.05)$.

radioactive esophagitis (grade 1 , grade 2), radiation pneumonitis (grade 1, grade 2), radiation dermatitis, and radioactive lung injury were seen in both the groups (Table 4), but there was no significant difference in radiotherapy-related adverse reactions between the two groups $(P>0.05)$.

\section{Discussion}

Lungs are radiation-sensitive organs and its tolerance dose is low. It is difficult to achieve the theoretical radical dose for lung cancer through conventional radiotherapy, as it induces the accelerated reproliferation of the remaining tumor and results in treatment failure. Therefore, it is very important and necessary to improve the radiotherapy techniques to improve the radiotherapy dose effects.

In recent years, radiotherapy technology has made great progress. Today, modern radiation techniques can substantially increase the radiation dose of tumor without causing substantial injury to the normal tissues, thus improving the tumor local control rate and the quality of life of the patients.

Stereotactic irradiation technology was initially used in clinical application in the early 1990s. ${ }^{9}$ Stereotactic radiation can maximally focus the radiation dose on the target area, improve the irradiation dose of tumor, and, meanwhile, reduce the dose to the surrounding normal tissue, thus reducing the radiation complications to a maximum extent.

Because of the heterogeneity of tumor cells, a larger fractionated dose is required for controlling the heterogeneity 
Table 2 Treatment effect

\begin{tabular}{|c|c|c|c|}
\hline Treatment effect & $\begin{array}{l}\text { Hypofractionated } \\
\text { group (\%) }\end{array}$ & $\begin{array}{l}\text { Conventional } \\
\text { fractionated group (\%) }\end{array}$ & $P$-value \\
\hline I-year OS rate & 87.0 & 63.0 & $P<0.05$ \\
\hline 2-year OS rate & 59.5 & 50.6 & $P>0.05$ \\
\hline I-year local failure survival rate & 95.2 & 72.8 & $P<0.05$ \\
\hline 2-year local failure survival rate & 88.9 & 48.5 & $P<0.05$ \\
\hline I-year local failure-free survival rate & 87.0 & 57.8 & $P<0.05$ \\
\hline 2-year local failure-free survival rate & 53.9 & 47.3 & $P>0.05$ \\
\hline Local failure rate & 13.0 & 40.7 & $P<0.05$ \\
\hline Distant metastasis rate & 21.7 & 44.4 & $P>0.05$ \\
\hline Median survival time & 34 months & 17.5 months & \\
\hline
\end{tabular}

Notes: Log-rank method was employed to compare survival rate between the groups. The survival rate at a certain time point was compared with $Z$-test; $Z>$ I.96 was statistically significant. Local failure rate and distant metastasis rate were tested by chi-square test; $P \leq 0.05$ was considered as statistical significance.

Abbreviation: OS, overall survival.

of tumor cells within a tumor. Studies on cell kinetics showed that the potential doubling time of lung cancer cell is longer than the average doubling time of other kinds of tumor cells, so lung cancer cells are suitable for hypofractionated irradiation. ${ }^{10}$ Hypofractionated radiotherapy delivers stronger lethality to tumor cells, reduces the accelerated reproliferation of the remaining tumor cells during the treatment, and shortens the treatment course. Hypofractionated stereotactic radiotherapy can improve the radiotherapy effect and could be the best choice for those lesions whose diameter is less than $5 \mathrm{~cm}$ or constitute metastatic lesions. However, the effect of parameters such as best single dose, total dose, and irradiation interval time needs to be further explored.

How to choose the best single irradiation fractionation dose, total dose, irradiation interval time, and fractional manner? Consideration should be given to all the factors to make the best treatment plan including the general condition of the patients, disease, Karnofsky score, lesion location, size, histological type, tolerance dose of the surrounding normal tissue, biological equivalent dose, and should also take into account whether the patient has received conventional external irradiation.

Our results showed that the treatment effect of hypofractionated radiotherapy was significantly better than the conventional fractionation radiotherapy and did not increase the toxic side effects. Hypofractionated radiotherapy significantly improved the treatment effect for advanced
NSCLC. The increased treatment effect may be related to high biological radiation dose, because hypofractionated radiotherapy showed significantly higher total biological equivalent dose. The biological equivalent dose ranges from 10,560 cGy to 11,520 cGy in this study.

Tumor local control rate is closely related to the total treatment time. Some mathematical models and studies indicated that the tumor local control rate increased in pace with the increasing irradiation dose in hypofractionated radiotherapy. ${ }^{11-14}$ It may be related to the short treatment course of hypofractionated radiotherapy which avoids the accelerated reproliferation and the repopulation of tumor cells. Prolonged overall treatment time will reduce the survival rate in lung cancer.

Studies have shown that the doubling time of NSCLC cells is 3-3.5 days, and accelerated repopulation of tumor cells appears in 3-4 weeks after the beginning of radiotherapy. ${ }^{15}$ Hypofractionated radiotherapy shows stronger tumor lethality and can effectively increase the tumor local control rate and shorten the treatment course, thus reducing the reproliferation of tumor cells. We used a dose of 5-8 Gy each time, three times a week. It generally took $2-3$ weeks to complete the treatment and obtain satisfactory results for NSCLC.

Currently, irradiation technology includes more precise techniques such as IMRT and IGRT derived from the traditional 2D radiotherapy techniques. Precise radiotherapy

Table 3 Position errors detected by volume CT $(x \pm s)$

\begin{tabular}{lllll}
\hline Direction & Before correction & After correction & After treatment & $P$-value \\
\hline Left-right & $0.32 \pm 0.14 \mathrm{~cm}$ & $0.22 \pm 0.11 \mathrm{~cm}$ & $0.14 \pm 0.11 \mathrm{~cm}$ & 0.088 \\
Anterior-posterior & $0.22 \pm 0.13 \mathrm{~cm}$ & $0.16 \pm 0.12 \mathrm{~cm}$ & $0.13 \pm 0.12 \mathrm{~cm}$ & 0.945 \\
Cranial-caudal & $0.28 \pm 0.22 \mathrm{~cm}$ & $0.19 \pm 0.22 \mathrm{~cm}$ & $0.13 \pm 0.13 \mathrm{~cm}$ & 0.778 \\
\hline
\end{tabular}

Note: ${ }^{a}$ One-way ANOVA.

Abbreviations: ANOVA, analysis of variance; $\mathrm{CT}$, computed tomography; $\mathrm{x} \pm \mathrm{s}$, mean \pm standard deviation. 
Table 4 Radiotherapy adverse reactions

\begin{tabular}{|c|c|c|c|}
\hline Adverse reactions & $\begin{array}{l}\text { Hypofractionated } \\
\text { group }\end{array}$ & $\begin{array}{l}\text { Conventional } \\
\text { fractionated group }\end{array}$ & $P$-value ${ }^{a}$ \\
\hline Radioactive esophagitis (grade I, grade 2) & $44.44 \%$ & $40.00 \%$ & $P>0.05$ \\
\hline Radiation pneumonitis (grade I, grade 2) & $44.44 \%$ & $40.00 \%$ & $P>0.05$ \\
\hline Radiation dermatitis & $11.11 \%$ & $20.00 \%$ & $P>0.05$ \\
\hline Blood toxicity & $22.22 \%$ & $30.00 \%$ & $P>0.05$ \\
\hline
\end{tabular}

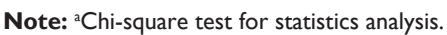

mainly embody in the control of three aspects of accuracy: precise positioning, precise planning, and precise treatment. Precise radiotherapy reduces not only the system errors but also the random errors at each step. IGRT is very important to ensure the accuracy of radiotherapy which includes precisely shooting the tumor, avoiding damage to normal tissue.

IGRT technology is very important to ensure all the conditions of irradiation treatment are the same as conditions of radiotherapy planning in real time. Real-time adjustment of position errors can greatly improve the accuracy of patient positioning and effectively reduce the errors. ${ }^{16-22}$ IGRT technology is definitely very helpful and contributes a lot to the accuracy of radiotherapy to an unprecedented level. The accuracy of irradiation is one of the key factors to increase the single irradiation dose and total irradiation dose of tumor and to reduce the irradiation on normal tissue and the side effects. IGRT helps in improving the treatment effect.

This study measured the position of target center changes in three dimensions by using Synergy system, and compared adjusted target center with the position of planning target center. At the same time, the treatment target center was rescanned by volume $\mathrm{CT}$ after the treatment, and the errors in lesion position were reanalyzed. This can accurately adjust the treatment target center to maintain consistency with the planning target center and further improve the treatment accuracy.

Our observations showed that all the positioning errors along the 3D directions were less than $5 \mathrm{~mm}$ and without statistical significance. The lesion position changes after the treatment also showed no statistical significance. The positioning errors were reduced after the adjustment but showed no statistical significance. The errors of target center were modified along the 3D directions by using volume CT technology to improve the precision of treatment.

The incidence of radiational esophagitis were observed in eight cases (44.44\%, level 1 and level 2) in group A and in four cases $(40 \%)$ in group B. The incidence of radiational pneumonitis was observed in eight cases (44.4\%) in group A and in four cases (40\%) in group B. No level 3 or 4 irradiation pneumonia was observed. The incidence of late radiational pneumonitis was found to be $10.1 \%(P>0.05)$.

Our results suggest that the adverse effects associated with the hypofractionated stereotactic radiotherapy are acceptable. These findings are consistent with other studies. ${ }^{23-27}$ Our results found that the incidence of short-term and long-term adverse effects of radiation toxicity and injury increased with the increase of the radiation dose. We determined that while planning fractionation dose, fractionation time, and the total dose, we should take the following factors into account: tumor size, tumor location, surrounding organs at risk, and the tolerated dose of the surrounding normal organs. We should not excessively emphasize on the high fractionation dose. The treatment efficiency and the treatment toxicity should be properly balanced. This study indicates that the image-guided hypofractionated stereotactic radiotherapy for peripheral NSCLC is feasible and further studies need to be carried out in this field.

In summary, hypofractionated stereotactic radiotherapy combined with IGRT for lung cancers can improve the therapeutic irradiation dose and treatment effects, shorten the total treatment time, and reduce the irradiation-related toxicity complications.

\section{Conclusion}

This study found that in hypofractionated group (group A), 1-year/2-year LFS and 1-year LFFS were significantly higher than in conventional fractionated group (group B) $(P<0.05)$. The local failure rate $(P<0.05)$ and distant metastasis rate $(P>0.05)$ of group A was lower than that in group B. The median survival time of group A was longer than that of group B. There was no significant difference in the incidence of complications between the two groups $(P>0.05)$. Compared with conventional fractionated radiation therapy, image-guided hypofractionated stereotactic radiotherapy in NSCLC received better treatment efficacy and achieved good tolerability. 


\section{Acknowledgments}

This study was supported by the National Natural Science Foundations of China (Juan Ren, 31201060/C0709; Juan Ren, 30973175/H1621; and Juan Ren, 81172490/H1621); Program for New Century Excellent Talents in University (Juan Ren, NCET-12-0440); Scientific and Technological Research Foundation of Shaanxi Province (Juan Ren, 2012K13-01-06); Scientific Research Foundation for the Returned overseas Chinese Scholars of State Education Ministry (Juan Ren, 0601-18920006); Research Foundation of Health Department of Shaanxi Province (Juan Ren, 2010D41); Qing Nian Jiao Shi Gen Zong Ji Hua of Xian Jiaotong University (The Fundamental Research Funds for the Central Universities) (Juan Ren, 2012).

\section{Author contributions}

All authors contributed toward data analysis, drafting and revising the paper and agree to be accountable for all aspects of the work.

\section{Disclosure}

The authors report no conflicts of interest in this work.

\section{References}

1. Mehta M, Scrimger R, Mackie R, Paliwal B, Chappell R, Fowler J. A new approach to dose escalation in non-small-cell lung cancer. Int J Radiat Oncol. 2001;49(1):23-33.

2. Trovo M, Giaj-Levra N, Furlan C, et al. Stereotactic body radiation therapy and intensity modulated radiation therapy induce different plasmatic cytokine changes in non-small cell lung cancer patients: a pilot study. Clin Transl Oncol. Epub 2015 Dec 21.

3. McBain CA, Henry AM, Sykes J, et al. X-ray volumetric imaging in image-guided radiotherapy: the new standard in on-treatment imaging. Int J Radiat Oncol Biol Phys. 2006;64(2):625-634.

4. Reporting Photon Beam Therapy (Supplement to ICRU Report 50), ICRU Report 62. Available from: http://www.icru.org/home/reports/ prescribing-recording-and-reporting-photon-beam-therapy-report-50. Accessed Jul 5, 2016.

5. Reporting Photon Beam Therapy (Supplement to ICRU Report 50), ICRU Report 62. Available from: http://www.icru.org/home/reports/ prescribing-recording-and-reporting-photon-beam-therapy-report-62. Accessed Jul 5, 2016.

6. Clivio A, Fogliata A, Franzetti-Pellanda A, et al. Volumetric-modulated arc radiotherapy for carcinomas of the anal canal: a treatment planning comparison with fixed field IMRT. Radiother Oncol. 2009;92(1): 118-124.

7. van't Riet A, Mak AC, Moerland MA, Elders LH, van der Zee W. A conformation number to quantify the degree of conformality in brachytherapy and external beam irradiation: application to the prostate. Int J Radiat Oncol Biol Phys. 1997;37:731-736.

8. Scorsetti M, Navarria P, Mancosu P, et al. Large volume unresectable locally advanced non-small cell lung cancer: acute toxicity and initial outcome results with rapid arc. Radiat Oncol. 2010;5:94.
9. Robertson JM, TenHaken RK, Hazuka MB, et al. Dose escalation for non-small cell lung cancer using conformal radiation therapy. Int J Radiat Oncol. 1997;37(5):1079-1085.

10. Rew DA, Wilson GD. Cell production rates in human tissues and tumours and their significance. Part II: clinical data. Eur J Surg Oncol. 2000;26(4):405-417.

11. Pedraza Muriel V. Hypofractionation in radiotherapy. Clin Transl Oncol. 2007;9(1):21-27.

12. Wein LM, Cohen JE, Wu JT. Dynamic optimization of a linear-quadratic model with incomplete repair and volume-dependent sensitivity and repopulation. Int J Radiat Oncol Biol Phys. 2000;47(4):1073-1083.

13. Jones B, Tan LT, Dale RG. Derivation of the optimum dose per fraction from the linear quadratic model. Br J Radiol. 1995;68(812):894-902.

14. Webb S. Optimum parameters in a model for tumour control probability including interpatient heterogeneity. Phys Med Biol. 1994;39(11): 1895-1914.

15. Fowler JF, Chappell R. Non-small cell lung tumors repopulate rapidly during radiation therapy. Int J Radiat Oncol. 2000;46(2):516-517.

16. Finnigan R, Lamprecht B, Barry $T$, et al. Inter- and intra-fraction motion in stereotactic body radiotherapy for spinal and paraspinal tumours using cone-beam CT and positional correction in six degrees of freedom. J Med Imaging Radiat Oncol. 2016;60(1):112-118.

17. Hou JZ, Zeng ZC, Wang BL, Yang P, Zhang JY, Mo HF. High dose radiotherapy with image-guided hypo-IMRT for hepatocellular carcinoma with portal vein and/or inferior vena cava tumor thrombi is more feasible and efficacious than conventional 3D-CRT. Jpn J Clin Oncol. 2016;46(4):357-362.

18. Simeonova-Chergou A, Jahnke A, Siebenlist K, et al. Automatically gated image-guided breath-hold IMRT is a fast, precise, and dosimetrically robust treatment for lung cancer patients. Strahlenther Onkol. 2016; 192(3);166-173.

19. Kilburn JM, Soike MH, Lucas JT, et al. Image guided radiation therapy may result in improved local control in locally advanced lung cancer patients. Pract Radiat Oncol. 2016;6(3):e73-e80.

20. Lee S, Yan G, Lu B, Kahler D, Li JG, Sanjiv SS. Impact of scanning parameters and breathing patterns on image quality and accuracy of tumor motion reconstruction in 4D CBCT: a phantom study. $J$ Appl Clin Med Phys. 2015;16(6):5620.

21. Nguyen NP, Karlsson UL. Editorial: image-guided radiotherapy for effective radiotherapy delivery. Front Oncol. 2015;5:253.

22. Elsayad K, Kriz J, Reinartz G, et al. Cone-beam CT-guided radiotherapy in the management of lung cancer: diagnostic and therapeutic value. Strahlenther Onkol. 2016;192(2):83-91.

23. Adebahr S, Collette S, Shash E, et al. An EORTC Phase II trial of stereotactic body radiotherapy for centrally located lung tumours: a clinical perspective. Br J Radiol. 2015;88(1051):20150036.

24. Boda-Heggemann J, Frauenfeld A, Weiss C, et al. Clinical outcome of hypofractionated breath-hold image-guided SABR of primary lung tumors and lung metastases. Radiat Oncol. 2014;9:10.

25. Janssen S, Dickgreber NJ, Koenig C, et al. Image-guided hypofractionated small volume radiotherapy of non-small cell lung cancer - feasibility and clinical outcome. Onkologie. 2012;35(7-8):408-412.

26. Ng AW, Tung SY, Wong VY. Hypofractionated stereotactic radiotherapy for medically inoperable stage I non-small cell lung cancer-report on clinical outcome and dose to critical organs. Radiother Oncol. 2008; 87(1):24-28.

27. Ohashi T, Takeda A, Shigematsu N, et al. Differences in pulmonary function before vs 1 year after hypofractionatedstereotactic radiotherapy for small peripheral lung tumors. Int J Radiat Oncol Biol Phys. 2005; 62(4):1003-1008. 
OncoTargets and Therapy

\section{Publish your work in this journal}

OncoTargets and Therapy is an international, peer-reviewed, open access journal focusing on the pathological basis of all cancers, potential targets for therapy and treatment protocols employed to improve the management of cancer patients. The journal also focuses on the impact of management programs and new therapeutic agents and protocols on

patient perspectives such as quality of life, adherence and satisfaction. The manuscript management system is completely online and includes a very quick and fair peer-review system, which is all easy to use. Visit http://www.dovepress.com/testimonials.php to read real quotes from published authors.

Submit your manuscript here: http://www.dovepress.com/oncotargets-and-therapy-journal 